\title{
Temperature dependence of exciton Auger decay process in Cuprous Oxide
}

\author{
Yingmei Liu, David Snoke \\ Department of Physics and Astronomy \\ University of Pittsburgh, Pittsburgh, PA 15260
}

(Dated:)

\begin{abstract}
We report theoretical and experimental study on Auger decay of excitons in $\mathrm{Cu}_{2} \mathrm{O}$ in a wide range of temperatures, from 2 Kelvin to 325 Kelvin, in single-photon surface excitation. We find that the Auger constant linearly increases with temperature above 100 Kelvin. This constant is important for experiments on Bose condensation of excitons.
\end{abstract}

PACS numbers: 


\section{INTRODUCTION}

There is a long history in investigating excitonic effects in naturally-grown high-quality $\mathrm{Cu}_{2} \mathrm{O}$, because of the inversion symmetry in $\mathrm{Cu}_{2} \mathrm{O}$ and the large binding energy of the excitons, $150 \mathrm{meV}$. The inversion symmetry forbids any direct dipole recombination of excitons which gives excitons long lifetime up to microsecond, and $150 \mathrm{meV}$ is equivalent to a temperature of 1740 Kelvin which allows excitons to exist at room temperature ${ }^{1}$. We are only interested in the lowest excitonic state, the "yellow" exciton series, consisting of electrons in the $\Gamma_{6}^{+}$level of the conduction band and holes in the $\Gamma_{7}^{+}$level of the valence band with 2.173 eV gap energy. By electron-hole exchange, the "yellow" excitons split into a triplet orthoexciton and a singlet paraexciton, which lies 12 meV lower ${ }^{1}$.

In the last ten years, there have been a lot of experiments done with excitons in $\mathrm{Cu}_{2} \mathrm{O}$, either with an intense surface excitation which creates a highly non-equilibrium exciton gas, or with an inhomogeneous strain which confines excitons in a harmonic potential trap with almost constant volume. One interesting topic among those experiments is the importance of the Auger non-radiative recombination process in Bose condensation of excitons. In the Auger process, two excitons collide, and one exciton ionizes, taking the energy of the other exciton which recombined. Some groups believe that the density-dependent Auger process is a barrier for Bose condensation of excitons, since it not only gives a severe limit in exciton density, but also heats up the exciton gas ${ }^{1.2 .3 .4}$, while others claim that the process is negligible ${ }^{5}$. Trauernicht et al. first gave strong evidences of an Auger process by trapping the excitons with an external stress ${ }^{6}$.

Our previous work $\frac{1}{\underline{\underline{T}}}$ has shown that the Auger recombination process is important and increases roughly as the square of the applied stress, but it left one question in doubt, why there is a very small Auger effect at stresses lower than 1.5 kbar at 2 Kelvin, while a strong Auger effect must occur in surface excitation with a negligible stress at higher temperatures, for example at room temperature. Does the surface play an important role in the Auger effect, or is the Auger effect temperature dependent? In this work, our theoretical and experimental results show that the Auger decay rate increases linearly with temperature, which answers the above question. 


\section{EXPERIMENT}

Excitons are created at one [110] surface of a $4.9 \times 3 \times 3 \mathrm{~mm}$ naturally-grown, high-quality $\mathrm{Cu}_{2} \mathrm{O}$ crystal with single-photon near-resonant excitation under negligible applied stress in the high temperature region, 115 325 Kelvin. An imaging lens collects exciton luminescence from the same [110] surface and focuses the light onto an entrance slit of a $\frac{1}{4}$-meter spectrometer which is connected with a CCD camera and a photomultiplier (PMT). The exciton population dynamics are examined by means of time-correlated single photon detection. This experimental approach has several advantages. First, in the high temperature region, paraexciton-to-orthoexciton conversion leads the excitons to reach thermal equilibrium quickly. Second, as pointed out by Warren and Wolfe ${ }^{2}$, the diffusion constant at high temperature is much smaller than that at low temperature, which allows a constant-volume measurement. Also, in these experiments, we use a cavity-dumped ultra-fast dye laser which has a long period, $260 \mathrm{~ns}$ between pulses. Therefore paraexcitons definitely reach thermal equilibrium with orthoexcitons. The bath temperature in the experiments is controlled by a homemade PID controller with an accuracy of \pm 2 Kelvin.

In $\mathrm{Cu}_{2} \mathrm{O}$, there are two possible single-photon transitions, a direct transition by emitting a photon, and a phonon-assisted transition by emitting a photon and an optical phonon. Because the indirect transition is allowed for all exciton states, the indirect phonon-assisted luminescence spectrum shows the energy distribution of excitons ${ }^{7}$. At high temperature, the direct single-photon transitions for orthoexcitons and paraexcitons are almost forbidden, while the phonon-assisted process depends weakly on temperature ${ }^{8}$. In Section 4, we will talk about phonon-assisted orthoexciton recombination processes, assisted by all possible optical phonons and acoustic phonons, although the orthoexciton creation process via a $\Gamma_{12}^{-}$longitudinal optical phonon is much stronger than other phonon-assisted orthoexciton creation processes 8 .

In the surface excitation, the absorption coefficient of $\mathrm{Cu}_{2} \mathrm{O}$ has a strong relation to bath temperature and laser photon energy ${ }^{9.10}$. We calculate absorption lengths of $\mathrm{Cu}_{2} \mathrm{O}$ for the input laser at $606 \mathrm{~nm}$ and for the orthoexciton luminescence at each temperature from Pastrnyak's report ${ }^{9}$ and J. B. Grun's paper ${ }^{10}$ on the absorption coefficient of $\mathrm{Cu}_{2} \mathrm{O}$, as shown in Figure 1. With a focused laser spot with radius of $120 \mu \mathrm{m}$, the orthoexciton has a volume of $\pi \times(120 \mu \mathrm{m})^{2} \times($ Absorption-length $)$, which is in the order of $10^{-6} \mathrm{~cm}^{-3}$. As we 
did in previous works ${ }^{1,8}$, we assume that one absorbed photon produces an exciton, since the laser is tuned close to the exciton resonance. At the highest laser power of $2.64 \mathrm{~nJ}$ per pulse, with 30\% absorbed in the crystal after considering all the optics involved, there are $10^{9}$ orthoexcitons created initially, which implies an initial orthoexciton density in the order of $10^{15} \mathrm{~cm}^{-3}$.

Because the absorption length at the orthoexciton luminescence line is of the order of a millimeter at high temperatures, which is comparable to the width of $\mathrm{Cu}_{2} \mathrm{O}$ sample, we have to add a correction factor to deduce the exciton density from the luminescence intensity at each temperature. The correction factor, $\mathrm{CF}$, is equal to the ratio between $I_{\text {detected }}$, the real detected luminescence intensity, and $I_{0}$, the ideal detected luminescence intensity without taking into account of the absorption of the luminescence in $\mathrm{Cu}_{2} \mathrm{O}$, by a Photomultiplier .

If we assume that one absorbed photon creates one exciton, the total number of excitons at a certain position $x$ in the sample, is proportional to the number of absorbed photons, $n_{x} \propto\left(e^{\frac{-x}{L_{1}}}\right)$, where $0 \leq x \leq L$ and $\mathrm{L}$, the width of $\mathrm{Cu}_{2} \mathrm{O}$, is 3 millimeter. $\mathrm{L}_{1}$ and $\mathrm{L}_{2}$ are the absorption lengths for the laser and orthoexciton luminescence at each temperature, respectively. Because of the absorption of the exciton luminescence in the sample, the number of the detected exciton from the position $x$ is proportional to $\left(e^{\frac{-x}{L_{2}}}\right)$. In other words, the total contribution to the luminescence from the position $x$ is proportional to $\left(e^{\frac{-x}{L_{1}}} e^{\frac{-x}{L_{2}}}\right)$.

Therefore, the detected luminescence intensity is proportional to the integration of the detected excitons over the whole length of the sample, and the correction factor is calculated as the following,

$$
\begin{array}{r}
C F=\frac{I_{\text {detected }}}{I_{0}}=\int_{0}^{L} e^{\frac{-x}{L_{1}}} e^{\frac{-x}{L_{2}}} d x \div \int_{0}^{L} e^{\frac{-x}{L_{1}}} d x \\
\Rightarrow C F=\frac{1}{L_{1}} \cdot \frac{1}{\frac{1}{L_{1}}+\frac{1}{L_{2}}} \cdot \frac{1-e^{-L\left(\frac{1}{L_{1}}+\frac{1}{L_{2}}\right)}}{1-e^{-\frac{L}{L_{1}}}}
\end{array}
$$

In principle, we should account for re-emission from the reabsorbed photons, but since the re-emission mostly goes in directions other than $\mathrm{x}$ (see inset of Figure 1), this will be a small correction.

\section{RESULTS AND DISCUSSIONS}

Figure 2 shows the typical orthoexciton photoluminescence intensity as a function of time after a short laser pulse, at two different temperatures, 150 and 325 Kelvin, and under three 
different pulse energies, $2.64 \mathrm{~nJ}, 2.64 / 2=1.32 \mathrm{~nJ}$ and $2.64 / 6=0.44 \mathrm{~nJ}$, multiplied by overall factors of 1, 2 and 6 respectively. At each temperature, the different decay times for different laser powers indicate that there is the density-dependent Auger process of excitons.

Our previous work ${ }^{1.8}$ presented coupled rate equations including the Auger decay rate, which explained well the data at 2 Kelvin under different stresses and laser pulse energies. However, because of phonon emission and absorption, the orthoexciton and paraexciton are well coupled in the high temperature region ${ }^{1}$, and therefore the coupled rate equations can be modified to the following:

$$
\begin{aligned}
& \frac{d n_{o}}{d t}=-\frac{n_{o}}{\tau_{o-p}}-A_{o} n_{o}^{2}+\frac{3}{8}\left(A_{o} n_{o}^{2}+A_{p} n_{p}^{2}\right)-\frac{n_{o}}{\tau_{o}}+\frac{n_{p}}{\tau_{p-o}}, \\
& \frac{d n_{p}}{d t}=\frac{n_{o}}{\tau_{o-p}}-A_{p} n_{p}^{2}+\frac{1}{8}\left(A_{o} n_{o}^{2}+A_{p} n_{p}^{2}\right)-\frac{n_{p}}{\tau_{p}}-\frac{n_{p}}{\tau_{p-o}} .
\end{aligned}
$$

In these equations, indies o and p stand for "orthoexciton" and "paraexciton", respectively, $A$ is the Auger decay constant, $\mathrm{n}$ is the exciton density, $\tau$ is the decay time or conversion time, and the indies o-p and p-o mean orthoexciton-paraexciton conversion and paraexcitonorthoexciton conversion, respectively. In each Auger process, two excitons collide and end up with one exciton recombining and the other exciton ionizing. Therefore, only the ionized exciton, half of the excitons participating in the Auger process, will be added back to exciton population. Because the orthoexciton is a triplet state and the paraexciton is a singlet state and spin is randomly selected in ionization, $\frac{3}{4}$ of the ionized excitons in the Auger process will be returned as orthoexcitons and $\frac{1}{4}$ of these excitons will be returned as paraexcitons. We have to consider inter-conversions between paraexcitons and orthoexcitons in the high temperature region, although only orthoexcitons are converted to paraexcitons at $2 \operatorname{Kelvin}{ }^{4}$. Because energy splitting between orthoexciton and paraexciton at the zone center is $12 \mathrm{meV}$, and orthoexciton and paraexciton reach thermal equilibrium rapidly at high temperature ${ }^{2}$, the conversion time in the inter-conversion mechanisms has a relationship,

$$
\tau_{p-o}=\tau_{o-p} \times e^{\frac{12 m e V}{K_{B} T}}
$$

All the data, under ten different temperatures and three different laser pulse energies at each temperature, are well fit with the above coupled equations, as shown on the solid lines of Figure 2 (a) and (b). The fit results imply that the Auger constant of the orthoexciton is equal to that of the paraexciton at each temperature. The results also show that the radiative lifetimes of paraexcitons and orthoexcitons are on the order of several hundred nanoseconds, 
and the inter-conversion time between paraexcitons and orthoexcitons is on the order of 0.1 nanosecond which is consistent with the report from Wolfe's group 2 and our previous results $\frac{1}{1}$. Because paraexcitons rapidly reach thermal equilibrium with orthoexcitons, in the fitting process we can assume that at all times after $\mathrm{t}=0.1 \mathrm{~ns}$,

$$
n_{p}(t)=n_{o}(t) \times e^{\frac{12 m e V}{K_{B} T}}
$$

An interesting result from the fitting is that the Auger constant of excitons linearly increases with temperature in the region from 115 Kelvin to 325 Kelvin, as shown in Figure 3. Several groups have studied the temperature dependence of the Auger decay process in $\mathrm{Cu}_{2} \mathrm{O}$, but the highest temperature they used is 77 Kelvinn 2,11 . Their works show that the Auger constant of orthoexcitons is almost independent of temperature. The above two results are not inconsistent, which is explained in detail in next section.

\section{PHONON-ASSISTED MECHANISM}

Kavoulakis and Baym ${ }^{11}$ introduced a model based on Fermi's Golden rule and $\mathbf{k} \cdot \mathbf{p}$ theory to understand the Auger recombination process with an optical phonon-assisted mechanism at low temperature. We follow their steps, but modify it with considerations of high temperature effects and all possible phonons in an Auger process, in which two excitons with momentum $\vec{K}$ and $\vec{P}$ collide, producing one ionized electron-hole pair with momenta $\vec{k}_{e}$ and $\vec{k}_{h}$, and absorbing or emitting a phonon.

In the phonon-assisted process, $\frac{\Gamma_{\text {Auger }}}{V}$, the Auger decay rate per unit volume is a function of $\Gamma_{K, P}$ and $f_{K}, f_{P}$, the decay rate and distribution functions of the two excitons, respectively:

$$
\frac{\Gamma_{\text {Auger }}}{V}=\frac{1}{V} \sum_{K, P} f_{K} f_{P} \Gamma_{K, P} \sim A n^{2}
$$

Where $\mathrm{A}$ is Auger constant for the exction and $\mathrm{n}$ is the density of the excitons.

From Fermi's Golden Rule, the decay rate of two excitons with momenta $\vec{K}$ and $\vec{P}$ is

$$
\begin{aligned}
\Gamma_{K, P}= & \frac{2 \pi}{\hbar} \sum_{k_{e}, k_{h}, Q}|M|^{2}\left(1-n_{c, k_{e}}\right)\left(1-n_{v, k_{h}}\right) \\
& \times\left[\left(n_{p h}+1\right) \delta\left(E_{K}+E_{P}-\hbar \omega_{Q}-\varepsilon_{c, k_{e}}-\varepsilon_{v, k_{h}}\right)\right. \\
& \left.+n_{p h} \delta\left(E_{K}+E_{P}+\hbar \omega_{Q}-\varepsilon_{c, k_{e}}-\varepsilon_{v, k_{h}}\right)\right]
\end{aligned}
$$


The first term in Equation 8 is for a process emitting a phonon with momentum $\vec{Q}$, while the second term is for a phonon absorption process by absorbing a phonon with momentum $\vec{Q}$. The term $n_{p h}=1 /\left(e^{E_{p h} /\left(k_{B} T\right)}-1\right)$ is the density of the phonon at temperature $\mathrm{T}$, and $k_{B}$ is Boltzmann's constant.

In the equations, based on $\mathbf{k} \cdot \mathbf{p}$ theory ${ }^{11}$, the matrix element of an optical phonon-assisted process, $\mathrm{M}_{\text {optical }}$, is given by the following equation,

$$
\begin{aligned}
M_{\text {optical }} & \approx 2^{7} \pi \frac{e^{2}}{\epsilon_{\infty} a_{B}} \frac{\hbar}{m} \times \frac{\left|\mathbf{P}_{v, c^{\prime}}\right|}{\left(\varepsilon_{c^{\prime}, 0}-\varepsilon_{v, 0}\right)} \times \frac{k_{e} a_{B}}{\left[1+\left(k_{e} a_{B}\right)^{2}\right]^{3}} \\
& \times\left(\frac{\hbar^{2} D_{\text {optical }}^{2}}{2 V \rho E_{\text {optical }}}\right)^{\frac{1}{2}} \frac{1}{\left(\varepsilon_{c, 0}-\varepsilon_{c^{\prime}, 0}\right)} \times \delta_{\vec{k}_{e}+\vec{k}_{h} \pm \vec{Q}, \vec{K}+\vec{P}} .
\end{aligned}
$$

where $\mathrm{D}_{\text {optical }}$ is the deformation potential for an optical phonon, $E_{\text {optical }}$ is the energy of the optical phonon, $\varepsilon_{c}$ and $\varepsilon_{c^{\prime}}$ are energies of the lowest conduction band $\Gamma_{6}^{+}$and the only odd parity conduction band $\Gamma_{8}^{-}$, respectively, $\varepsilon_{v}$ is the energy of the valence band $\Gamma_{7}^{+}, P_{v, c^{\prime}}$ is the matrix element of the momentum operator between the conduction and valence band at zone center, $\rho$ is the mass density of $\mathrm{Cu}_{2} \mathrm{O}, \epsilon_{\infty}$ is the dielectric constant, and $\mathrm{a}_{B}$ is the exciton Bohr radius.

In an acoustic phonon assisted process, $k_{\text {necessary }}$ the necessary momentum for an acoustic phonon is to satisfy with energy and momentum conservations between the lowest conduction band and the only odd parity conduction band, that is to say, $k_{n e c e s s a r y}=\left(\varepsilon_{c}-\varepsilon_{c^{\prime}}\right) /(\hbar \nu)$ $=1.515 \times 10^{9} \mathrm{~cm}^{-1}$. In the discussion, $\nu$ is the acoustic phonon speed. However, $k_{\max }$, the maximum momentum of an acoustic phonon, is equal to $\frac{\pi}{a_{L}}=7.375 \times 10^{7} \mathrm{~cm}^{-1}$ because it is restricted to be in the first Brillouin zone, where $a_{L}$ is the lattice constant of $\mathrm{Cu}_{2} \mathrm{O}$. Therefore there is no possible process assisted by the acoustic phonon and from now on all discussions will only consider the process assisted by the optical phonon.

As mentioned by Kavoulakis ${ }^{11}, E_{K}, E_{k_{e}}$, and $E_{k_{h}}$, the energy of an exciton with momentum $\vec{K}$, the energy of an electron in the conduction band, and the energy of a hole in the valence band, respectively, are given by $\stackrel{11}{ }$,

$$
\begin{aligned}
& E_{K}=E_{\text {gap }}+\frac{\hbar^{2} K^{2}}{2 m_{\text {exciton }}} \\
& E_{k_{e}}=E_{\text {gap }}+\frac{\hbar^{2} k_{e}^{2}}{2 m_{e}} \\
& E_{k_{h}}=\frac{\hbar^{2} k_{h}^{2}}{2 m_{h}}
\end{aligned}
$$


Because the momenta of excitons, $\vec{K}$ and $\vec{P}$, are determined by exciton thermal motion, we can make a reasonable assumption ${ }^{11}$,

$$
\vec{K}, \vec{P} \ll \vec{k}_{e}, \vec{k}_{h}
$$

By substituting Equation (9) through (13) into Equation (8), $\Gamma_{K, P}$ the decay rate of two excitons in the phonon-assisted process is equal to

$$
\begin{aligned}
\Gamma_{K, P}^{\text {honon-assisted }}= & \frac{2^{14} \pi^{3} e^{4} \mu \hbar}{\epsilon_{\infty}^{2} a_{B}^{2} V m^{2} E_{\text {optical }} \rho} \times \frac{D_{\text {optical }}^{2}}{\left(\varepsilon_{c, 0}-\varepsilon_{c^{\prime}, 0}\right)^{2}} \times \frac{\left|\mathbf{P}_{c^{\prime}, v}\right|^{2}}{\left(\varepsilon_{c^{\prime}, 0}-\varepsilon_{v, 0}\right)^{2}} \\
& \times \frac{\left(k_{e} a_{B}\right)^{2}}{\left[1+\left(k_{e} a_{B}\right)^{2}\right]^{6}} \times\left[1+\frac{2}{e^{E_{\text {optical }} / k_{B} T}-1}\right]
\end{aligned}
$$

The integration is restricted to the first Brillouin zone. All constant parameters used in the calculation are listed in Table 1. The optical deformation potentials and $\left|\mathbf{P}_{c, v}\right|^{2}$ are estimated from fitting of experimental data.

Therefore, by substituting Equation (14) into Equation (7), we get $A_{\text {phonon-assisted }}$, the phonon-assisted Auger constant, in the unit of $\mathrm{cm}^{3} / \mathrm{ns}$,

$$
A_{\text {phonon-assisted }} \simeq 3.698 \times 10^{-17} \times\left[1+\frac{2}{e^{160 / T}-1}\right]+1.298 \times 10^{-17} \times\left[1+\frac{2}{e^{217 / T}-1}\right] .
$$

Only two optical phonons, $\Gamma_{12}^{-}$and $\Gamma_{15}^{-}$, make important contributions.

We plot Equation 15, a theoretical prediction of the exciton Auger constant assisted by all possible phonons from 2 to 325 Kelvin, and our experimental Auger recombination constants in the high temperature region ( $115 \sim 325$ Kelvin ) in Figure 3, which shows that our experimental data in the high temperature region can be well fit by the theoretical prediction of the Auger constant from the phonon-assisted mechanism.

At low temperatures, where the phonon energy is much smaller than the thermal energy $\mathrm{k}_{B} \mathrm{~T}, \mathrm{n}_{p h}$ the density of the phonon is much less than 1 . Therefore only the phonon emission process is important and phonon absorption process is negligible. We can approximate:

$$
\begin{aligned}
\Gamma_{K, P}= & \frac{2 \pi}{\hbar} \sum_{k_{e}, k_{h}, Q}\left|M_{p h}^{2}\right|\left(1-n_{c, k_{e}}\right)\left(1-n_{v, k_{h}}\right) \\
& \times \delta\left(E_{K}+E_{P}-\hbar \omega_{Q}-\varepsilon_{c, k_{e}}-\varepsilon_{v, k_{h}}\right) \quad(\text { Low T) }
\end{aligned}
$$

After substituting Equation (16) into Equation (7), we find that the Auger decay constant is weakly dependent on temperature when the temperature is lower than 77 Kelvin, which 
is shown in Fig. 3 and also consistent with the reports from Warren et $a l^{2} \stackrel{2}{ }$ and Kavoulakis et al. 2.11. $^{2}$.

Therefore, we can explicitly state our conclusion on the temperature dependence of the Auger recombination constant of excitons as the following: the Auger constant is weakly dependent on temperature when the temperature is below 77 Kelvin, and linearly increases with temperature in the high temperature region.

To verify our model, we make two comparisons. The first comparison is between a reported Auger constant from Wolfe's group ${ }^{2}$ and our theoretical prediction at 77 Kelvin, which are consistent with each other, though our theoretical prediction is about 4 percent larger, as shown in Figure 4. The other comparison is made between the experimental data and our theoretical model at 2 Kelvin. Our theoretical result indicates that the Auger constant does not continuously decrease to zero at zero stress at 2 Kelvin, but becomes a constant, $4.996 \times 10^{-17} \mathrm{~cm}^{3} / \mathrm{ns}$, which is comparable to the experimental data at 1.5 kbar at the same temperature reported in the previous work $\frac{1}{}$, as shown in Fig. 4 also. The comparison implies several things. First, the Auger constant has a negligible stress dependence when the stress is lower than $1.5 \mathrm{kbar}$. Second, the two results are consistent, although the theoretical prediction from our phonon-assisted mechanism is about 9 percent larger than the extrapolated data. Third, the surface doesn't play an important role in the Auger effect. Since the 4 to 9 percent discrepancy between experimental data and theoretical prediction is in the acceptable experimental error range, our modified phononassisted mechanism may be a good model for the Auger recombination rate of excitons in both low and high temperature regions.

\section{CONCLUSIONS}

From the above theoretical and experimental studies, we may say that in surface excitations, the Auger recombination constant linearly increases with temperature at high temperatures, and weakly depends on temperature in the low temperature region, which is

consistent with our modified model of phonon-assisted Auger recombination. However, we do not have a model for the stress dependence. 
Acknowledgements. This work has been supported by CRDF-MRDA Award No. MP2-3026 and NSF Award No. DMR-0102457. We thank S. Denev for his contribution on the PID temperature controller. Samples of $\mathrm{Cu}_{2} \mathrm{O}$ was obtained from P. J. Dunn of the Smithsonian Institute.

1 S. Denev and D. W. Snoke, Phys. Rev. B 65, 085211 (2001).

2 J. T Warren, K. E O’Hara, and J. P. Wolfe, Phys. Rev. B 61, 8215 (2000).

3 K. E O'Hara, J. R. Gullingsrud, and J. P. Wolfe, Phys. Rev. B 60, 10872 (1999).

4 G. M. Kavoulakis, Gordon Baym, and J. P. Wolfe, Phys. Rev. B 53, 7227 (1996).

5 A. Jolk, M. Jorger, and C. Klingshirn, Phys. Rev. B 65, 245209 (2002).

6 D. P. Trauernicht, J. P. Wolfe, and A. Mysyrowicz, Phys. Rev. B 34, 2561 (1986).

7 V. C. Negoita, Ph.D. thesis, University of Pittsburgh, (2001).

8 D. W. Snoke and V. Negoita, Phys. Rev. B 61, 2904 (2000).

9 I. Pasternyak, Phys. Status Solidi 1, 888 (1959).

10 J. B. Grun, M. Sieskind et S. Nikitine, J. Phys. Chem. Solids, Vol. 19, 189 (1961).

11 G. M. Kavoulakis and Gordon Baym, Phys. Rev. B 54, 16625 (1996).

12 D. W. Snoke, D. Braun, and M. Cardona, Phys. Rev. B 44, 2991 (1991).

13 David R. Lide, "Handbook of Chemistry and Physics ", 77th edition (1996-1997)

14 B. K. Ridley, "Quantum Processes in Semiconductors ", 2nd edition (1988)

15 Moskalenko and Snoke, "Bose-Einstein Condensation of Excitons and Biexcitons ", Cambridge University Press (2000) 


\begin{tabular}{|c|c|c|}
\hline Parameter & Value & References \\
\hline$\epsilon_{\infty}$ & 6.46 & 11 \\
\hline$\rho$ & $6 \mathrm{gram} / \mathrm{cm}^{3}$ & 13 \\
\hline$\nu$ & $4.5 \times 10^{5} \mathrm{~cm} /$ second & 12 \\
\hline$m_{e}$ & $m_{e}^{0}$ & 4 \\
\hline$m_{h}$ & $0.7 m_{e}^{0}$ & 4 \\
\hline$m_{e}^{0}$ & $9.1 \times 10^{28} \mathrm{gram}$ & 14 \\
\hline$a_{B}$ & $7 \times 10^{-8} \mathrm{~cm}$ & 10 \\
\hline$a_{L}$ & $4.26 \times 10^{-8} \mathrm{~cm}$ & 10 \\
\hline$D_{\Gamma_{12}^{-}}$ & $1.10 \pm 0.01 \mathrm{eV} / \AA$ & this work \\
\hline$D_{\Gamma_{15}^{-}}$ & $0.75 \pm 0.01 \mathrm{eV} / \AA$ & this work \\
\hline$\left|\mathbf{P}_{c^{\prime}, v}\right| / \hbar$ & $0.55 \pm 0.01 \AA-1$ & this work \\
\hline$\varepsilon_{c^{\prime}}-\varepsilon_{c}$ & $0.449 \mathrm{eV}$ & 11 \\
\hline$\varepsilon_{c^{\prime}}-\varepsilon_{v}$ & $2.622 \mathrm{eV}$ & 11 \\
\hline$\Gamma_{12}^{-}$ & $13.8 \mathrm{meV}$ & 15 \\
\hline$\Gamma_{15}^{-}$ & $18.7 \mathrm{meV}$ & 15 \\
\hline
\end{tabular}

TABLE I: Constant parameters used in the Auger decay rate calculation. 


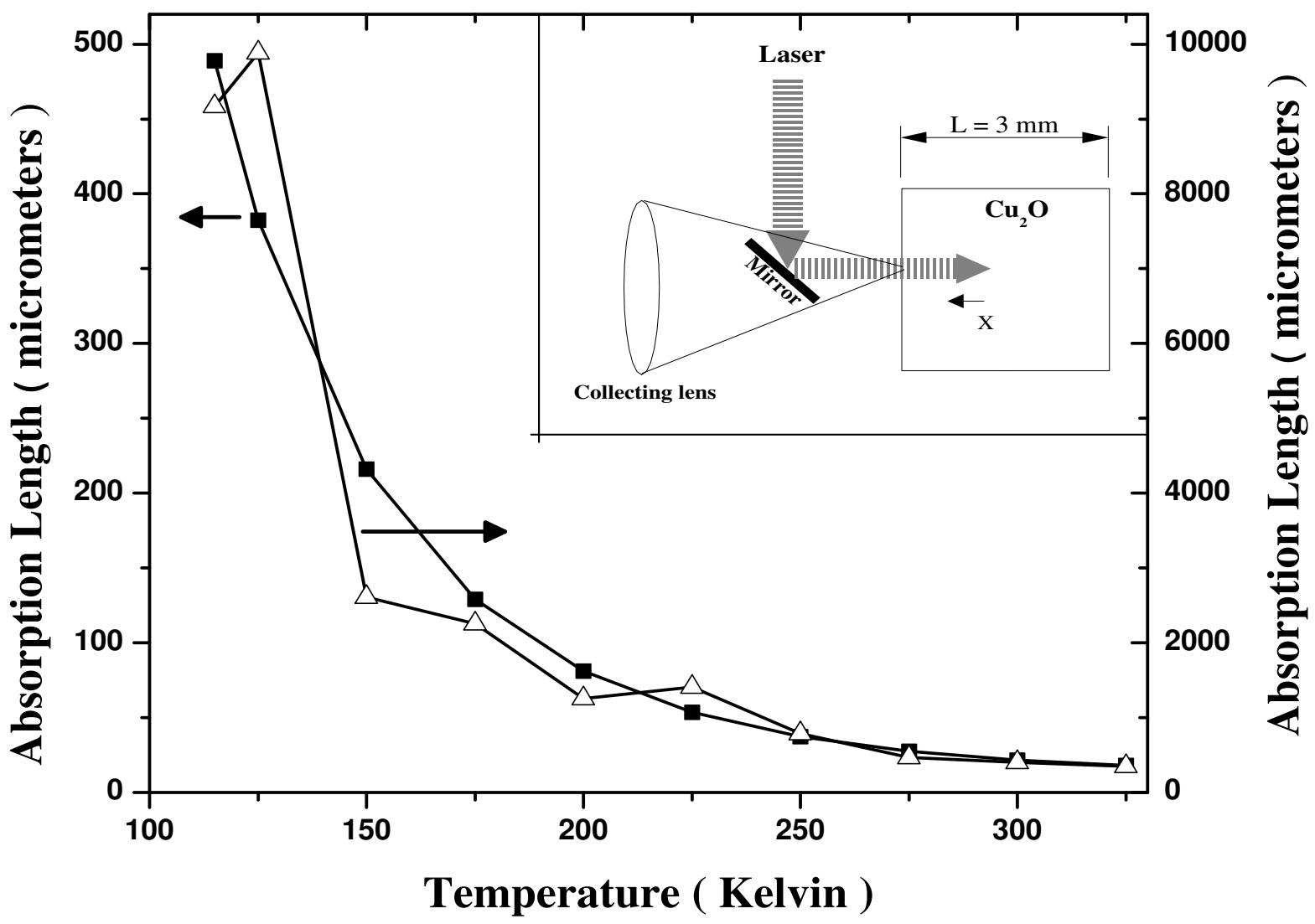

FIG. 1: Absorption lengths of the input laser at $606 \mathrm{~nm}$ ( black squares ), and the exciton luminescence ( open triangles ) in $\mathrm{Cu}_{2} \mathrm{O}$ at each temperature in the high temperature region. Inset: Schematic of the experimental set-up in this work. 
(a)

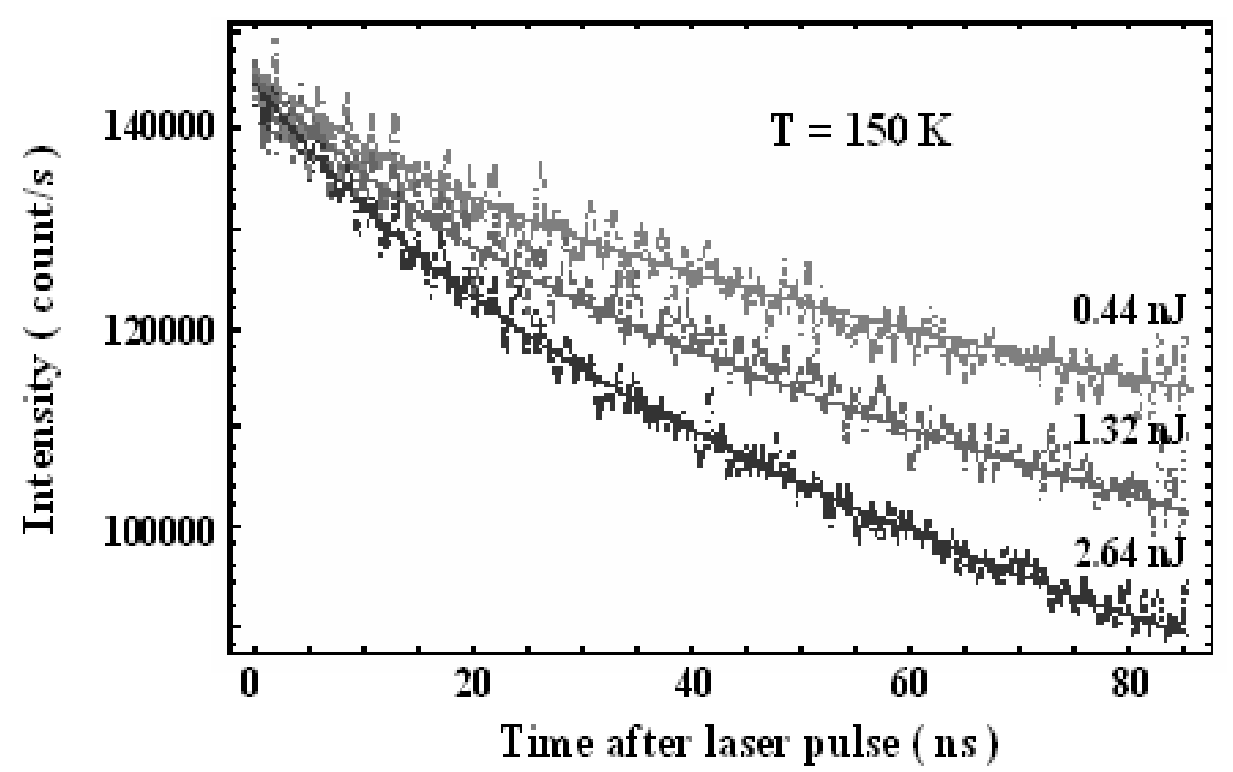

(b)

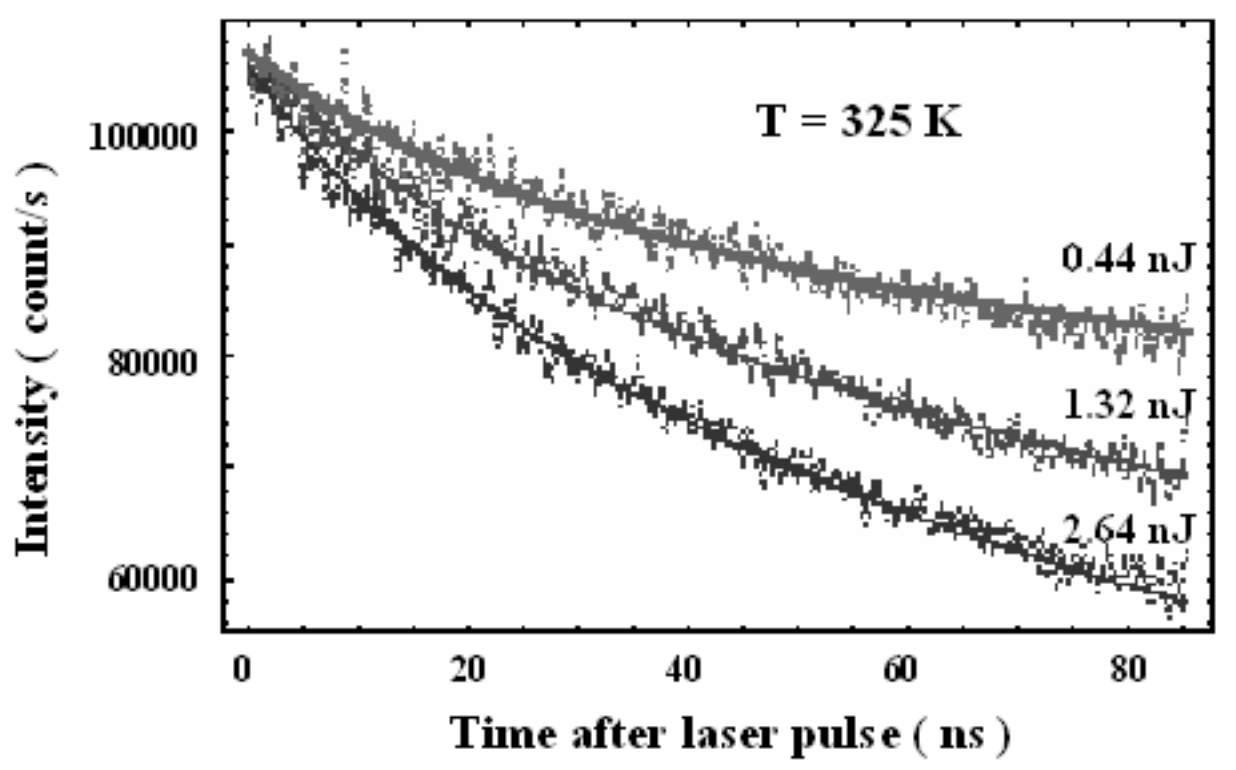

FIG. 2: (a) Orthoexciton luminescence decay at $\mathrm{T}=150$ Kelvin for three different pulse energies, $2.64 \mathrm{~nJ}, 1.32 \mathrm{~nJ}$ and $0.44 \mathrm{~nJ}$, as shown in the figure. Dots: Phonon-assisted orthoexciton luminescence intensity as a function of time after a short laser pulse. Solid lines: fit to the coupled rate equations discussed in the text. (b) Orthoexciton luminescence decay, as in (a), but at $\mathrm{T}=325$ Kelvin. 


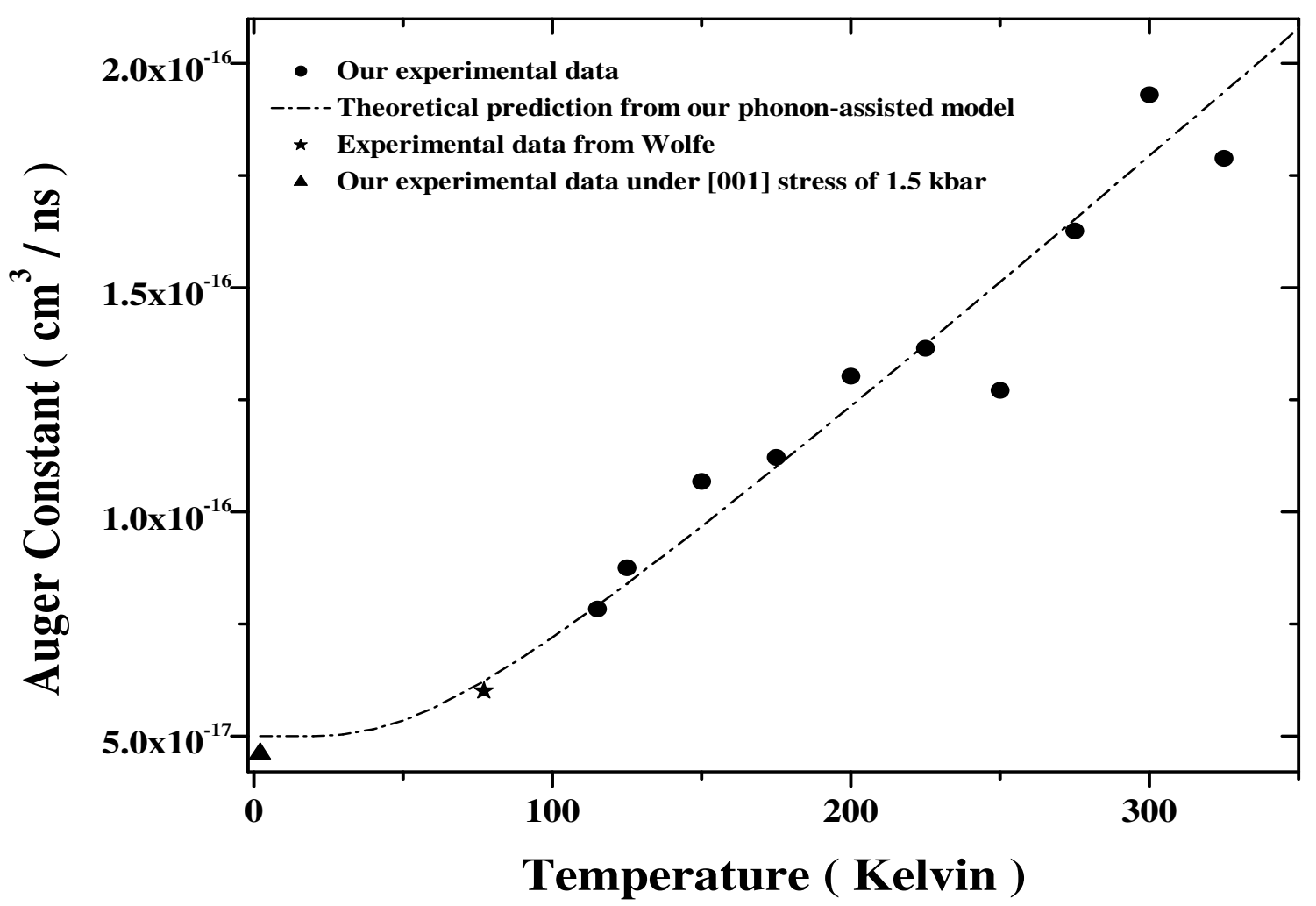

FIG. 3: Predicted and experimental Auger constants of the excitons as a function of temperature in the high and low temperature regions. 


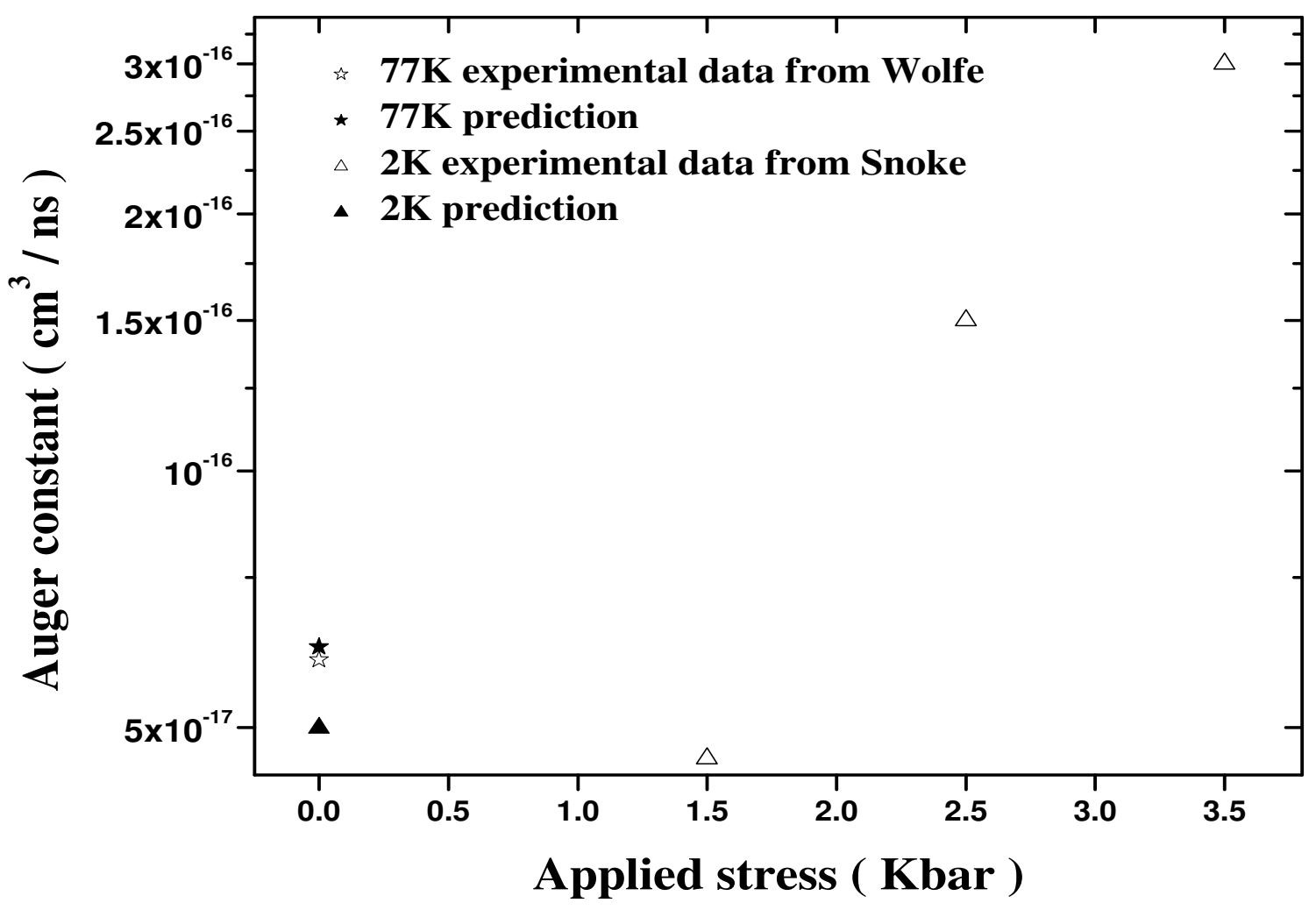

FIG. 4: Stress and temperature dependence of the orthoexciton Auger constant. Open triangles: data reported by Snoke et al $\frac{1}{\underline{1}}$ at 2 Kelvin for three different stresses, 1.5, 2.5 and 3.5 kbar. Open stars: data reported by Wolfe et al $\mathrm{s}^{2}$ with negligible stress at 77 Kelvin. Black triangles and black stars are predictions from our theoretical model with negligible stress at 2 Kelvin and 77 Kelvin, respectively. 